\title{
Ultra Cold Neutrons: Determination of the Electric Dipole Moment and Gravitational Corrections Via Matter Wave Interferometry
}

\author{
E. Abdalla and M. S. Hussein \\ Instituto de Física, Universidade de São Paulo, C. P. 66318, 05315-970, São Paulo, SP, Brazil \\ Received on 29 December, 2004
}

\begin{abstract}
We propose experiments using ultra cold neutrons which can be used to determine the electric dipole moment of the neutron itself, a well as to test corrections to gravity as they are foreseen by string theories and KaluzaKlein mechanisms.
\end{abstract}

Matter Wave Interferometry Devices (MWID) are aimed at testing one of the important pilars of quantum mechanics, namely wave-like interference of matter. These devices use the idea that quantum mechanical interference results in diffraction patterns of the intensity, which critically depend on the relative phase of the interfering waves. This allows the extraction of useful information about the phase generating, refractive-diffractive medium through which the wave propagates.

There are two main questions we are concerned with here, which appear in the context of grand unification and superstring theories. The first is that within grand unified theories with supersymmetry there are several plausible contributions to the electric dipole moments of eletrons and neutrons [1, 2], a problem known as the SUSY CP problem. CP violation is very little known, and the violating parameters, especially the neutron and the eletron electric dipole moments should be tested.

In fact, the Standard Model (SM) is an extremely efficient theory in what concerns experience today, but it is widely believed that there exists new physics beyond it and any extension of the SM, especially in the case it contains supersymmetry (SSM), which is also widely believed to be certain, foresees $\mathrm{CP}$ violation, since it is necessary to explain, from the observational point of view, the baryon asymmetry of the Universe [3].

In the case of the neutron electric dipole moment the relevant CP-violating terms are flavour blind. The contribution from the Kobayashi-Maskawa matrix arises at three loop level, and the experimental bound is [4]

$$
d_{n} \leq 6.3 \times 10^{-26} e \times \mathrm{cm} .
$$

The second question we deal with here concerns a specific point of string theories, which is the fact that they require the existence of further dimensions [5-7] which can be both of infinite [8-10] or finite $[11,12]$ size. This leads to corrections of Newton's gravity law at very small distances.

An experimental confirmation (or denial) of the above facts lies in the heart of the question whether grand unified theories with supersymmetry and string theories are part of physics or are just mathematically well defined objects beyond physical reality.

In a recent publication [14], an electric field-induced phase was measured with great precision for atomic waves. We propose that the same idea may be used to extract the dipole moment of the neutron, and test the behaviour of gravity for very short distances.
The first idea is to use Ultra-Cold Neutrons (UCN) of a velocity of $v_{n} \simeq 5 \mathrm{~m} / \mathrm{s}$, which pass through a splitter (crystal) before they actually interact with the electric field of an appropriate capacitor. The idea of using ultracold neutrons in order to probe properties of elementary particles has already been advocated [13]. When a neutron moves in a potential, $U(x)$, the phase of its wave function can be written as (considering this potential much smaller than its kinetic energy)

$$
\Delta \phi \approx-\frac{1}{\hbar v_{n}} \int U(x) d x
$$

where the limits of the integral are determined by the length of the phase generating apparatus. If $U(x)$ is a constant, then

$$
\Delta \phi \approx-\frac{1}{\hbar v_{n}} U L
$$

Therefore, the interference of the two split neutron waves, one of which affected by (3), would behave as

$$
I_{n} \approx A+B \cos \left(\phi_{0}+\Delta \phi \cos \theta\right),
$$

where $\theta$ is the angle between the parallel to the propagationin-medium wave, and that of the screen.

As the neutron wave traverses the region of strong electric field it feels an interaction which has the form

$$
U=\vec{d}_{n} \cdot \vec{\varepsilon}-\mu_{n}\left(\frac{v_{n}}{c} \varepsilon\right)-\frac{\alpha_{n} \varepsilon^{2}}{2},
$$

where the term $\left(-\frac{v_{n}}{c} \varepsilon\right)$ is the induced magnetic field owing to the motion of the particle through the electric field $\varepsilon$. The third term corresponds to the neutron stark effect interaction where the electric polarizability $\alpha_{n}$ enters into the picture. Of course, at the very slow velocities we are considering, gravity effects are relevant. These, however can be eliminated by a proper calibration of the experiment through a measurement the matter interference when the electric field is switched off.

From the numbers shown in the table we anticipate that the third term in Eq. (5) is much smaller than the first two, even for the reasonably strong field considered, namely $e \varepsilon \sim \frac{100 \mathrm{KeV}}{\mathrm{cm}}$. In so far as the competition between the electric and magnetic interaction of the neutron is concerned, we have evaluated both terms using the values of $\mu_{n}$ and $d_{n}$ given in the table, a neutron velocity of $5 \mathrm{~m} / \mathrm{s}$ and the above cited value of $\varepsilon$. We find

$$
\frac{d_{n}}{\mu_{n} \frac{v_{n}}{c}} \approx 10^{-4}
$$




\begin{tabular}{|c|c|}
\hline \multicolumn{2}{|c|}{ Table: fundamental constants } \\
\hline Neutron mass & $m_{n}=1.67 \times 10^{-27} \mathrm{Kg}$ \\
\hline Magnetic dipole moment & $-1.91 \times \frac{e \hbar}{2 m_{p} c^{2}}$ \\
\hline Neutron Lifetime & $\tau=(889.1 \pm 2.1) s$ \\
\hline Electric polarizability & $\alpha=(0.98 \pm 0.20) \times 10^{-42} \mathrm{~cm}^{3}$ \\
\hline Charge & $(-0.4 \pm 1.1) \times 10^{-21} e$ \\
\hline Electric Dipole moment & $d_{n} \leq 6.3 \times 10^{-26} e \times c m$ \\
\hline Newton constant & $G=6.67 \times 10^{-11} \mathrm{~m}^{3} / \mathrm{Kg} \mathrm{s}^{2}$ \\
\hline
\end{tabular}

It is clear that the measurement of the phase shift of the two beams of neutrons will simply be sensitive to $\mu_{n}$.

In order to remove the effect of the magnetic interaction to first order we suggest using an external magnetic field that is opposite to the induced one,

$$
\vec{B}_{e x t}=\frac{\vec{v}_{n}}{c} \times \vec{\varepsilon}
$$

This field is quite weak and can be easily attained. In fact, due to the smallness of (6), there is no practical way of cancelling such a term with the corresponding one in (5) to the desired accuracy, since the velocity $v_{n}$ itself is defined to an accuracy of a few percent. However, if we allow for several total reflections, $N$, of the neutrons from mirrors placed at the ends of the capacitor this would on the one hand enhance the electric interaction as the phase becomes

$$
\Delta \phi_{e}=-\frac{N U L}{\hbar v_{n}}=\frac{N}{\hbar v_{n}} d_{n}(e V) \frac{L}{a},
$$

with a still very small (almost cancelled) magnetic interaction which reverses sign for a neutron traversing the cylinder back and forth. In eq. (8), $\mathrm{eV}$ is the voltage across the capacitor and $a$ is the distance between the plattes. Taking $\frac{L}{\alpha}=10^{4}(L=1 \mathrm{~m}$, $a=1 \mathrm{~mm}$ ) and using for $N \simeq 1001$, (odd number of collisions guarantees that the neutrons come out at the other end of the capacitor), we find (for $d_{n} \sim 0.6 \times 10^{-25} \mathrm{ecm}$ )

$$
\Delta \phi_{e} \simeq 2 \times 10^{-3} \mathrm{rad} \simeq 0.1^{\circ}
$$

a measurable shift in the diffraction maxima seen on the screen. For $N \sim 1000$ and an external $B$ field given with an accuracy of $1 \%$ the difficulty related to the smallness of the electric dipole moment, (6) is overcome (with the cancellation of the velocity dependent term for back and forth travels) and we have a result with a $10 \%$ confidence. In fact angular shifts of the order of $10^{-3}$ have already been measured in another quantal wave mechanical problem involving Mott-type oscillation in the elastic scattering of two slowleads ions [14]. The purpose of these measurements was the verification of the possible existence of long-range multigluon exchange interaction (colour van der Waals force) which were predicted to exist in several theories [15]. Therefore an enhancement of one order of magnitude in the neutron electric dipole moment limit can be obtained.

We should emphasize that, in $\Delta \phi_{e}$, we have na very small number, $d_{n}\left(\simeq 10^{-25} \mathrm{ecm}\right)$ multiplying a very large number, $N\left(\frac{L}{a}\right) \frac{1}{\hbar v_{n}}$. This latter can be made larger with a suitable changes in the macroscopic variables that define it. For a time of flight approximately equal to the neutron lifetime, $N$ can be as large as 5000 .

We now pass to the second proposal. As mentioned, according to string theory, world is multidimensional. The question of how do the extra dimensions behave is however not fixed, at least from the theoretical side, since there are several possibilities, namely the extra dimensions might be of the size of the Planck lenght [5], they can have a finite submilimetric size in order to unify the Planck and Standard Model scales [11] or they can be infinite with a nontrivial warp in the extra dimensions [9]. It is an important experimental problem to prove these extra dimensions by proposing new situations where they can leave an experimental imprint $[10,16]$. A recent proposal in this direction was given in [17].

We thus propose to use the slow neutrons to measure the new contributions to Newtons potential. We suppose that since gravitational fields are weak, the metric, either $3+1$ dimensional, or 4+1-dimensional is well described in terms of a potential as $g_{00}=-1+\frac{2 U_{g}}{c^{2}}$, with $U_{g}$ given by the solution of the Poisson equation. We propose that the neutrons are colimated into a small hollow cilinder. In accordance with $3+1$ dimensional Newton law the potential due to a line of matter with linear density $\lambda$, located at the $z$-axis from $z=0$ to $z=L$ is

$$
U_{g}(x)=G \lambda \ln \frac{(L-z)+\sqrt{r^{2}+(L-z)^{2}}}{-z+\sqrt{r^{2}+z^{2}}}
$$

where $r$ is the distance to the $z$-axis. We shall suppose that for a hollow cilindes the potential inside the cilinder is given by the above with $r=r_{0}$ being the internal radius.

For a 4+1-dimensional world the expression is different, and we suppose that it is effective only for distances smaller than a typical size of the extra dimension $\xi \sim 0.1 \mathrm{~mm}$. Therefore, inside the hollow cylinder we subtract the $3+1$ dimensional contribution of a slice $z \varepsilon[z-\xi, z+\xi]$ and add a 4+1-dimensional contribution of a slice $z \varepsilon[z-\xi, z+\xi]$, obtaining

$$
\begin{aligned}
U_{g}(z) & \simeq G \lambda\left\{\ln \frac{(L-z)+\sqrt{r^{2}+(L-z)^{2}}}{-z+\sqrt{r^{2}+z^{2}}}+\frac{2 \xi}{r}\right. \\
\left.-\ln \frac{\xi+\sqrt{r^{2}+\xi^{2}}}{-\xi+\sqrt{r^{2}+\xi^{2}}}\right\} & \\
& \simeq G \lambda\left\{\ln \frac{4 z(L-z)}{r^{2}}+\frac{2 \xi}{r}-\ln \frac{\xi+\sqrt{r^{2}+\xi^{2}}}{-\xi+\sqrt{r^{2}+\xi^{2}}}\right\}
\end{aligned}
$$

The order of magnitude of the effect on a slow neutron is not negligible, that is

$$
\Delta \phi_{g}=N \frac{U_{g} m_{n} L}{\hbar v} \simeq N \frac{G \lambda m_{n} L}{\hbar v}=6 \times 10^{-3}=0.3^{o}
$$

for a cylinder of linear density $\lambda \sim 0.03 \mathrm{Kg} / \mathrm{m}$, and $N \sim 1000$, which is comparable to the value of $\Delta \phi_{e}$ in eq. (9) for the electric dipole measurement. The above numbers are compatible with a thin cylinder of radius $1 \mathrm{~mm}$. For a cylinder of $0.3 \mathrm{~mm}$, 
quite comparable to the presumed size of the extra dimension, we still get a measurable result [14]. Notice that $N \sim 5000$ is still compatible with the neutron lifetime.
Acknowledgement: This work was partially supported by FAPESP and CNPQ, Brazil.
[1] Y. Y. Keum and Otto C. W. Kong, Phys. Rev. D 63, 113012 (2001), hep-ph/0101113; Phys. Rev. Lett. 86, 393 (2001), hep$\mathrm{ph} / 0004110$.

[2] Yosef Nir, 27th SLAC Summer Institute on Particle Physics: CP Violation in and Beyond the Standard Model (SSI 99), Stanford, California, 7-16 Jul 1999, *Trieste 1999, Particle physics* 165243, hep-ph/9911321.

[3] A. D. Sakharov JETP Lett. 5, 24 (1967); G. R. Farrar and M. E. Shaposhnikov Phys. Rev. D50 (1994) 774; P. Huet and E. Sather Phys. Rev. D 51, 379 (1995).

[4] P. G. Harris et al Phys. Rev. Lett. 82, 904 (1999).

[5] Michael B. Green, J. H. Schwarz, Edward Witten, Superstring Theory, Cambridge Monographs On Mathematical Physics, 1987.

[6] J. Polchinski String Theory Cambridge Univ. Pr., 1998.

[7] J. Scherk Rev. Mod. Phys. 47, 123 (1975).

[8] Petr Horava and Edward Witten, Nucl. Phys. B 460, 506 (1996), hep-th/9510209.

[9] Lisa Randall and Raman Sundrum Phys. Rev. Lett. 83, 4690
(1999).

[10] Elcio Abdalla, Adenauer Casali, and Bertha Cuadros-Melgar, Nucl. Phys. B 644, 201 (2002), hep-th/0205203.

[11] Ignatios Antoniadis, Nima Arkani-Hamed, Savas Dimopoulos and G. R. Dvali Phys. Lett. B 436, 257 (1998), hep$\mathrm{ph} / 9804398$.

[12] Lisa Randall and Raman Sundrum Phys. Rev. Lett. 83, 3370 (1999).

[13] O. Bertolami and F. M. Nunes Class. Quantum Grav. 20, L61 (2003).

[14] A. C. C. Villari et al Phys. Rev. Lett. 71, 2551 (1993).

[15] M. S. Hussein, C. L. Lima, M. P. Pato, and C. A. Bertulani Phys. Rev. Lett. 65, 839 (1990).

[16] M. S. Hussein. R. Lichtenthäler, M. P. Pato, and C. A. Bertulani Braz. J. Phys. 27, (1997).

[17] A. Frank, P. Van Isacker, and J. Gomez-Camacho Phys. Lett. B 582, 15 (2004). 